\title{
Searches for high mass Higgs bosons with the ATLAS detector*
}

\author{
S. Angelidakis ${ }^{\mathrm{a}}$, on behalf of the ATLAS collaboration \\ ${ }^{a}$ National and Kapodistrian University of Athens, Greece
}

\begin{abstract}
Recent results from searches for heavy Higgs bosons, using the ATLAS detector at the CERN LHC are presented. The searches use proton-proton collision data collected during the second running period of the LHC (LHC Run-II), at a centre-of-mass energy of $13 \mathrm{TeV}$, corresponding to an integrated luminosity of $3.2 \mathrm{fb}^{-1}$. The results are interpreted in a range of scenarios, including theories beyond the Standard Model of particle physics, and extend the upper limits set during LHC Run-I toward higher mass regions.
\end{abstract}

Keywords: Higgs, ATLAS, LHC

\section{Introduction}

The discovery of a new particle with a mass of about $125 \mathrm{GeV}$ by the ATLAS [1] and CMS [2] experiments at the Large Hadron Collider (LHC) [3], which was announced in July 2012 [4, 5], provided, by all experimental evidence so far, an excellent candidate for the Higgs boson predicted by the Standard Model (SM) of particle physics [6, 7, 8]. One important remaining question is whether or not the newly discovered particle is part of an extended scalar sector as postulated by various extensions to the SM such as the two-Higgsdoublet model (2HDM) [9] and the Minimal Supersymmetric Standard Model (MSSM) [10]. These models predict additional Higgs bosons, motivating searches at masses other than $125 \mathrm{GeV}$. The sections that follow report separate searches with the ATLAS detector for additional, heavy Higgs-like bosons. Unless specified otherwise, the results presented have been produced using the data collected with ATLAS during 2015, at $\sqrt{s}=13 \mathrm{TeV}$, which correspond to an integrated luminosity of $3.2 \mathrm{fb}^{-1}$.

${ }^{*}$ Talk given at the 19th High-Energy Physics International Conference in Quantum Chromodynamics (QCD 16), 4 - 8 July 2016, Montpellier - FR.

Email address: Stylianos. Angelidakis@cern.ch (S. Angelidakis)

\section{2. $H \rightarrow Z Z$}

The various final states of the $H \rightarrow Z Z$ decay mode are studied in search of a neutral, CP-even, spin-0 Higgs boson $H$ with SM-like couplings. Both narrow (i.e. narrower than the experimental resolution) and large-width hypotheses are examined for the Higgs boson.

\section{1. $Z Z \rightarrow \ell^{+} \ell^{-} q \bar{q}$}

In the final state with two leptons and two quarks, a pair of oppositely charged leptons (electrons or muons) and a pair of high- $p_{\mathrm{T}}$ jets are searched for [11, 12]. The analysis is divided into two main channels; the resolved analysis attempts to reconstruct and identify two separate hadronic jets from the $Z \rightarrow q q$ decay whereas the merged analysis implements boosted techniques, evaluating the jet-substructure, in order to identify a $Z \rightarrow q q$ decay that has been reconstructed as a single largeradius (large- $R$ ) jet. The latter is expected when the resonance mass is significantly higher than the $Z$ boson mass. For the resolved analysis, candidate events are further categorised based on the number of $b$-tagged jets in order to take advantage of the different signalto-background ratios in these categories. No significant excess of data is found over the SM prediction in the spectrum of the invariant mass of the two leptons and the one/two jet system. Upper limits on the product 


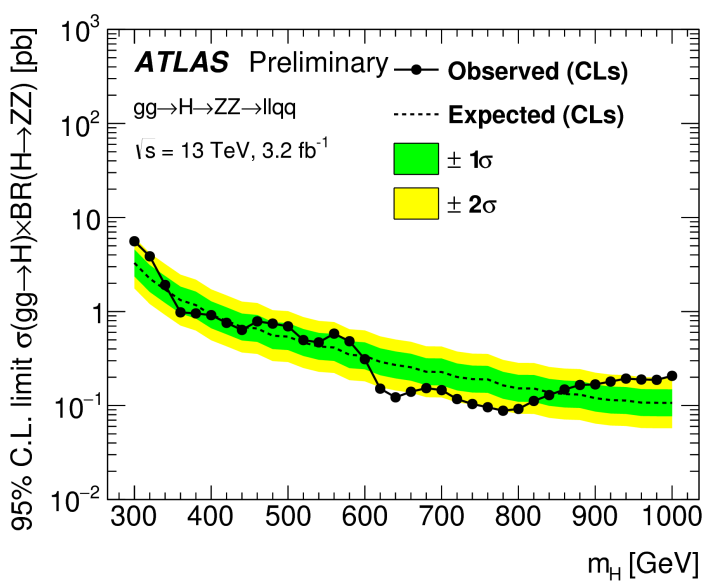

Figure 1: Observed (solid line) and median expected (dashed line) 95\% CL limits on the product of the production cross section times the branching ratio to a pair of $Z$ bosons, derived with the $\ell \ell q q$ channel, for a narrow-width Higgs boson, as a function of the hypothesized Higgs boson mass $m_{H}[11]$. The green and yellow bands correspond to $\pm 1 \sigma$ and $\pm 2 \sigma$ intervals for the expected exclusion limit, respectively.

of the Higgs boson production cross section times the branching ratio to a pair of $Z$ bosons are estimated at 95\% confidence level (CL) as shown in Figure 1 for the intermediate Higgs boson mass range $300 \mathrm{GeV}-1 \mathrm{TeV}$.

\section{2. $Z Z \rightarrow v \bar{v} q \bar{q}$}

The final state with two neutrinos and two quarks is studied in the context of both $H \rightarrow Z Z$ and $H \rightarrow W Z$ decay modes [13]. Both cases consider a $Z$ boson decaying to neutrinos, giving rise to large missing transverse momentum $\left(E_{\mathrm{T}}^{\mathrm{miss}}\right)$, while the other boson ( $W$ or $Z$ ) decays hadronically. The search is performed in the mass interval from $1 \mathrm{TeV}$ to $3 \mathrm{TeV}$. As in the previous case, for high mass states, the decay products of the hadronically decaying vector boson are expected to appear as a single jet in the detector, therefore, the candidate is reconstructed using boosted techniques. The discriminating variable used is an estimator of the $Z Z$ invariant mass known as the transverse mass $\left(m_{\mathrm{T}}\right)$ of the di-jet and $E_{\mathrm{T}}^{\mathrm{miss}}$ system. No significant deviation from the expected background is observed in the data. The estimated exclusion limits are presented in Figure 2

\section{3. $Z Z \rightarrow \ell^{+} \ell^{-} \ell^{\prime} \ell^{\prime}$}

The Higgs boson decay to four leptons provides good sensitivity due to its high signal-to-background ratio $(\mathrm{S} / \mathrm{B})$. A search for a heavy, scalar resonance $S$ is performed in the final states: $4 \mu, 2 \mu 2 e, 2 e 2 \mu, 4 e$, where the first lepton pair is the one with invariant mass closest to the $Z$ boson mass [14]. Although the relatively

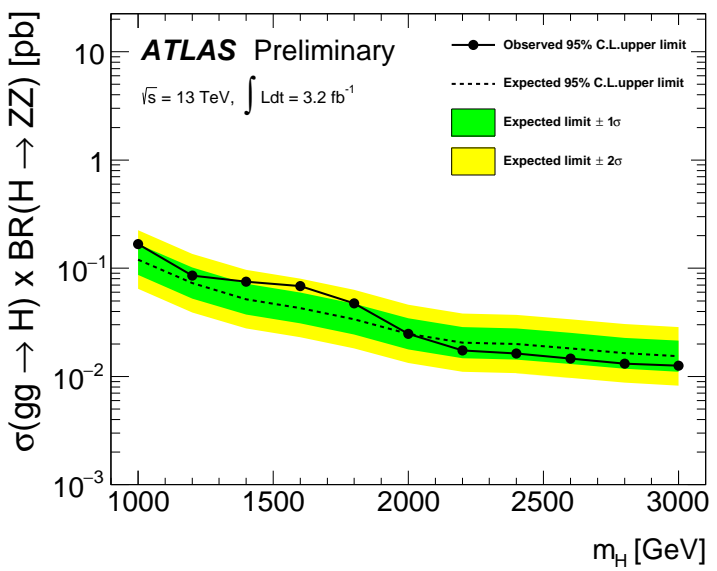

Figure 2: $95 \%$ CL upper limits on the product of the production cross section times the branching ratio to a pair of $Z$ bosons, derived with the $v v q q$ channel, for a narrow-width Higgs boson, as a function of the hypothesized Higgs boson mass [13].

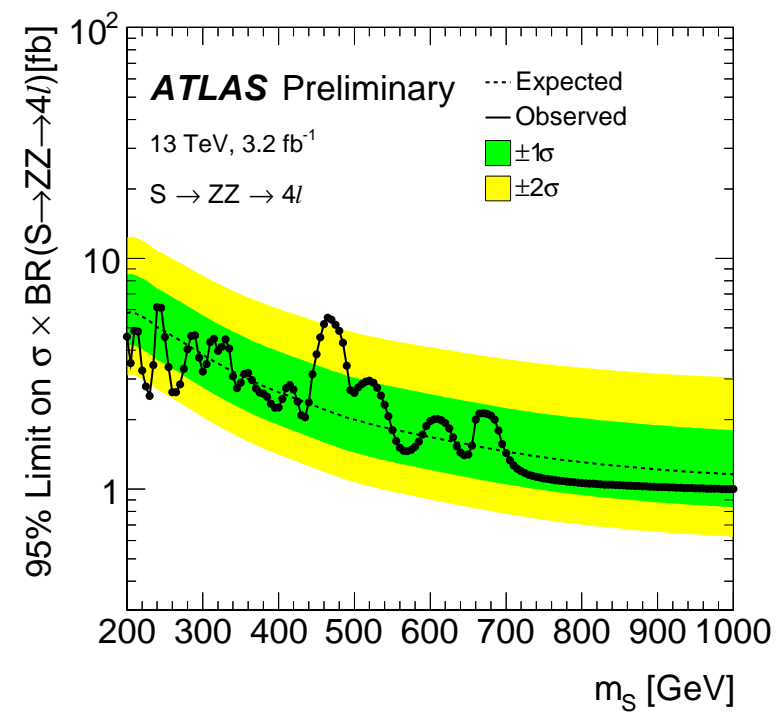

Figure 3: 95\% CL upper limits on $\sigma \times B R\left(S \rightarrow Z Z^{*} \rightarrow 4 \ell\right)$, derived with the $4 \ell$ channel, for a narrow-width resonance, as a function of the hypothesized resonance mass [14].

small branching ratio of $Z Z \rightarrow \ell^{+} \ell^{-} \ell^{\prime} \ell^{\prime}-$ compared to $Z Z \rightarrow \ell^{+} \ell^{-} q \bar{q}$ and $Z Z \rightarrow \ell^{+} \ell^{-} v_{\ell} \bar{v}_{\ell}$ renders the latter channels more sensitive for large scalar boson masses $\left(m_{S} \gtrsim 500 \mathrm{GeV}\right)$, the $4 \ell$ channel still improves the overall sensitivity. The search is performed in the mass interval $200 \leq m_{S} \leq 1000 \mathrm{GeV}$. No significant excess of the data is observed in the $m_{S}$ spectrum. The estimated 95\% CL upper limits on $\sigma \cdot B R\left(S \rightarrow Z Z^{*} \rightarrow 4 \ell\right)$ are presented in Figure 3 


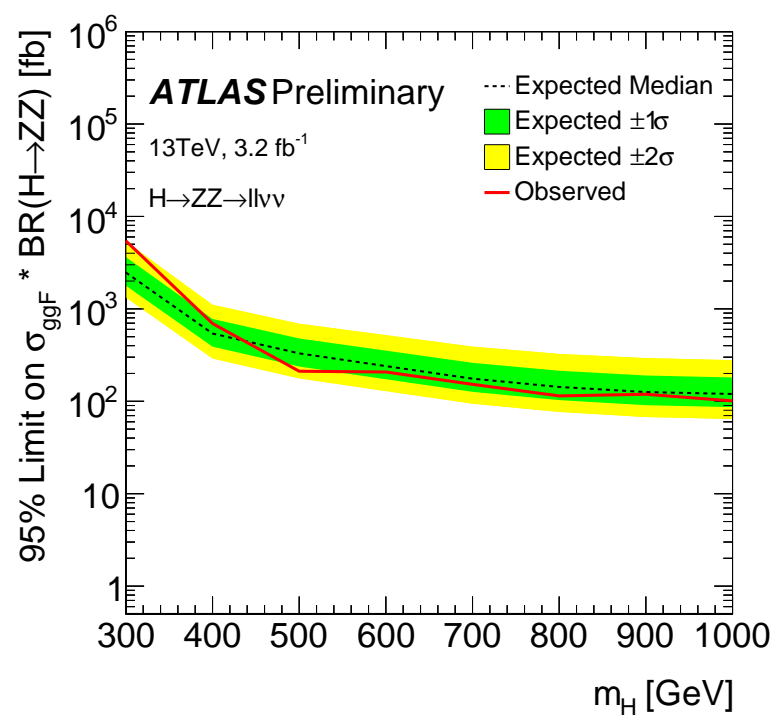

Figure 4: 95\% CL upper limits on $\sigma(p p \rightarrow X) \times B R(X \rightarrow Z Z)$, derived with the $\ell \ell v v$ channel, for a narrow-width resonance produced via gluon-gluon fusion, as a function of the hypothesized Higgs boson mass [15].

\section{4. $Z Z \rightarrow \ell^{+} \ell^{-} v_{\ell} \bar{\nu}_{\ell}$}

The final state with to leptons and two neutrinos is studied using events with large $E_{\mathrm{T}}^{\text {miss }}$ and a $Z$ boson candidate decaying into $e^{+} e^{-}$or $\mu^{+} \mu^{-}$[15]. The search covers the Higgs boson mass interval from $300 \mathrm{GeV}$ up to $1 \mathrm{TeV}$ using the transverse mass, obtained by combining $E_{\mathrm{T}}^{\mathrm{miss}}$ and the momentum of the $Z$ boson candidate, to discriminate a possible signal peak above the background from SM processes. The observed data are found to be consistent with the SM prediction and upper limits are set on the product of the production cross section times the branching ratio to a pair of $Z$ bosons, as illustrated in Figure 4

\section{3. $H \rightarrow W W$}

Two final states of the $H \rightarrow W W$ decay mode are investigated, $H \rightarrow W W \rightarrow \ell v \ell v$ and $H \rightarrow W W \rightarrow \ell v q q$ $(\ell=e, \mu)$ [16]. In the $H \rightarrow W W \rightarrow \ell v \ell v$ analysis, separate categories for zero, one, and at least two jets are defined. The zero- and one-jet categories are primarily sensitive to the gluon-gluon fusion production mode whereas higher jet multiplicity is used to identify candidates produced by vector boson fusion. For the reconstruction of this final state, only pairs of differentflavour leptons are considered. The discriminating variable used is the transverse mass, $m_{\mathrm{T}}$, of the selected leptons and $E_{\mathrm{T}}^{\mathrm{miss}}$ system.

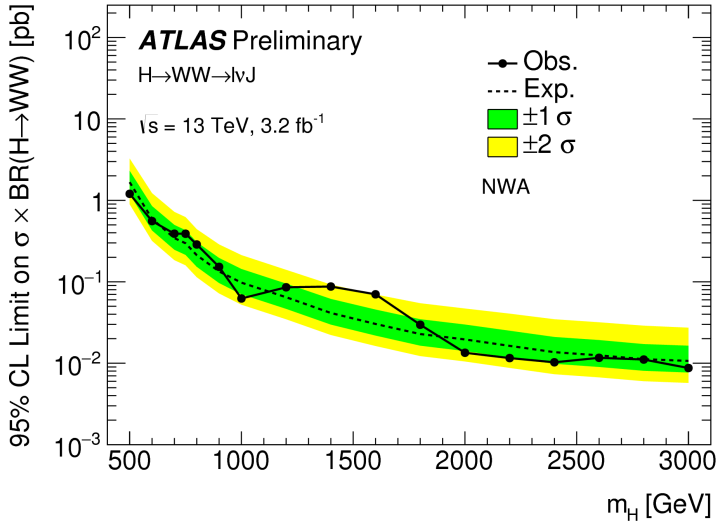

Figure 5: 95\% CL upper limits on the product of the production cross section of a narrow-width Higgs boson times the branching ratio to a pair of $W$ bosons, as a function of the hypothesized Higgs boson mass [16].

In the $H \rightarrow W W \rightarrow \ell v q q$ analysis, the $q q$ final state is reconstructed using boosted techniques due to the large boost acquired by the $W$ bosons from the massive Higgs boson decay. The discriminating variable used is the invariant mass of the reconstructed $W W$ pair, which can be estimated by imposing the $W$ boson mass constraint on the selected lepton and $E_{\mathrm{T}}^{\mathrm{miss}}$ system in order to recover the $z$-component of the neutrino's momentum. Since no significant excess of data is found above SM predictions, exclusion limits are estimated as shown in Figure 5 .

\section{4. $H \rightarrow \gamma \gamma$}

The search of the $\gamma \gamma$ final state is optimized for a spin-0 particle of mass larger than $200 \mathrm{GeV}$ [17]. A restricted kinematic range is used for the photon selection, taking advantage of the isotropic distribution of the decay products in the centre-of-mass frame of the new particle. The background is estimated by fitting the diphoton invariant mass distribution to an analytical function and the invariant mass of the di-photon system, $m_{\gamma \gamma}$ is scanned in search of an excess, modelled by a spin-0 resonance convolved with the experimental resolution. The mass range covered is $200-2000 \mathrm{GeV}$, where there are enough events to constrain the background shape, and for width values up to $10 \%$ of the mass of the hypothesized particle.

By varying both the mass and the decay width, the most significant deviation from the background-only hypothesis is observed at $m_{\gamma \gamma} \sim 750 \mathrm{GeV}$ with a local significance of 3.9 standard deviations. The global 


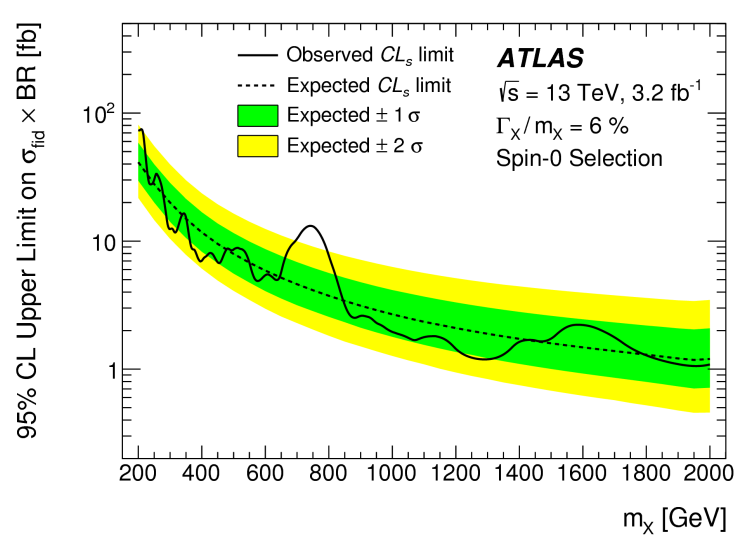

Figure 6: $95 \%$ CL upper limits on the fiducial cross section of a narrow-width, spin- 0 particle, derived with the $\gamma \gamma$ channel, as a function of the hypothesized particle mass [17].

significance is estimated to be 2.1 standard deviations. Limits on the production cross section times branching ratio to two photons are presented in Figure 6.

\section{5. $X \rightarrow Z \gamma$}

In the search for a heavy, scalar resonance decaying to a $Z$ boson and a photon, both leptonic and hadronic decay modes of the $Z$ boson are considered [18]. The analysis is optimized accordingly to select events in which the $Z$ boson decays to a pair of opposite charge electrons or muons, using lepton triggers (leptonic analysis, $\ell \ell \gamma)$, and events in which the $Z$ boson decays to hadrons (hadronic analysis, jet $+\gamma$ ), using single-photon triggers. This allows the use of the offline detector information to identify boosted $Z$ bosons from the merged di-jet cluster, reconstructed as a single, large- $R$ jet. The observed data are found to be consistent with the SM predictions, therefore, the statistical analysis sets $95 \%$ CL upper limits on the production cross section times the branching ratio to a $Z$ boson and a photon, as illustrated in Figure 7 .

\section{6. $H / A \rightarrow \tau \tau$}

A neutral (CP-even or CP-odd) Higgs bosons of the MSSM, decaying to a pair of $\tau$ leptons, is searched for in the mass range $200 \mathrm{GeV}-1.2 \mathrm{TeV}$ [19]. The Higgs boson is assumed to decay to a $\tau^{+} \tau^{-}$pair with at least one $\tau$ lepton decaying hadronically. Higgs boson production via gluon-gluon fusion or in association with a $b$-quark is considered, with the latter mode dominating at large $\tan \beta$ values. The observed data are found to

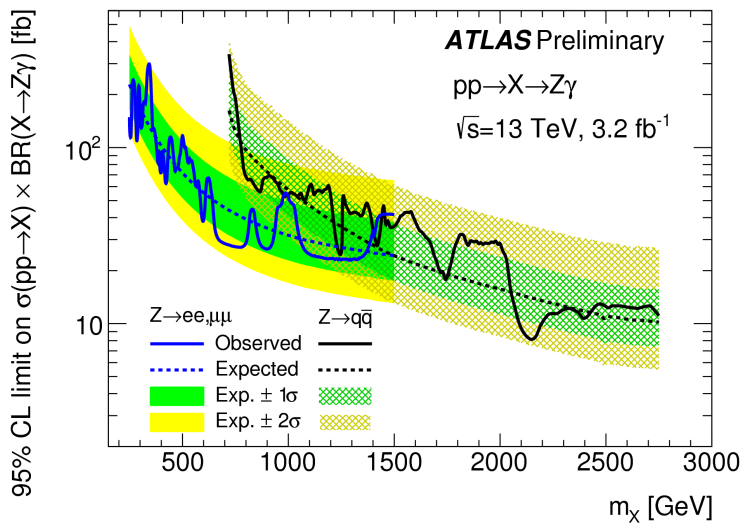

Figure 7: 95\% CL upper limits on the product of the production cross section times the branching ratio for the decay to a $Z$ boson and a photon, of a narrow-width scalar boson $X$, as a function of the hypothesized boson mass [18]. The black and blue lines correspond to the limits set with the jet $+\gamma$ and the $\ell \ell \gamma$ final state, respectively.

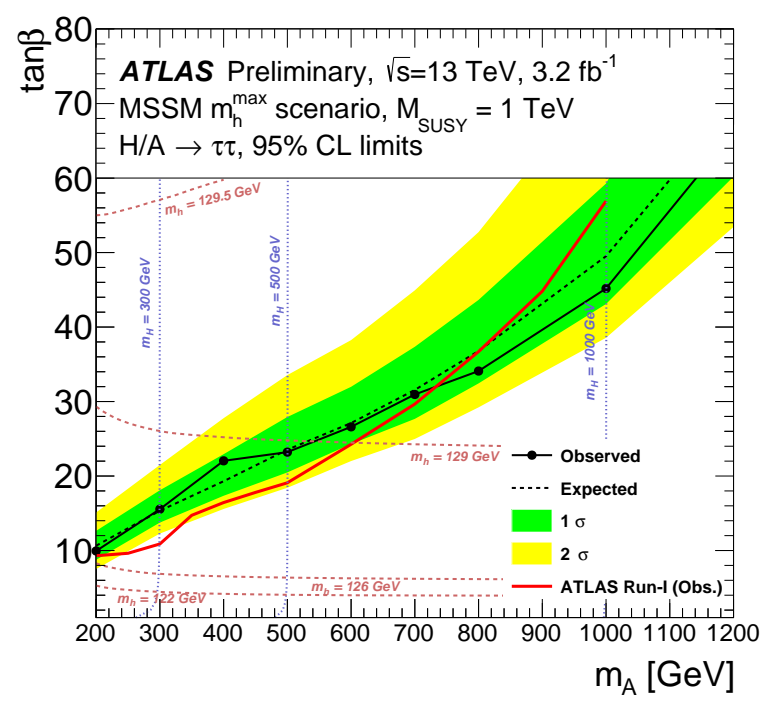

Figure 8: 95\% CL upper limits on $\tan \beta$ as a function of $m_{A}$ for the combination of the $\tau_{\text {lep }} \tau_{\text {had }}$ and the $\tau_{\text {had }} \tau_{\text {had }}$ channels in the $m_{h}^{\max }$ MSSM scenarios [19]. For comparison, the observed limits from LHC Run-I results are shown as a continuous red line. Dashed lines corresponding to constant $m_{h}$ and $m_{H}$ values are also shown in red and blue colour, respectively.

be consistent with the SM prediction, hence, results are given in terms of upper limits on the product of the production cross section times the branching ratio to $\tau^{+} \tau^{-}$. The results are interpreted in a range of MSSM scenarios (an example is shown in Figure 8) and improve the limits set by previous analyses, based on data collected at $\sqrt{s}=8 \mathrm{TeV}$, in the mass range $m_{A}>700 \mathrm{GeV}$. 


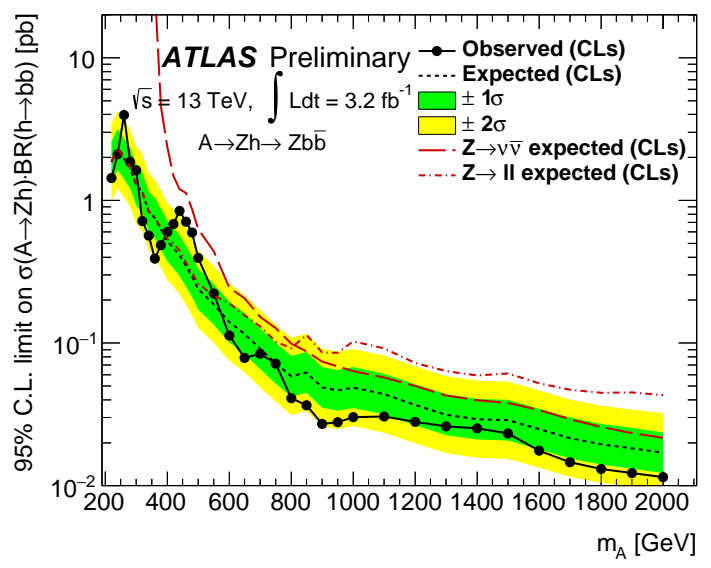

Figure 9: 95\% CL upper limits on $\sigma(p p \rightarrow A) \cdot B R(A \rightarrow Z h) \cdot B R(h \rightarrow$ $b \bar{b}$ ) for pure gluon-gluon fusion production. The long-dashed (finedotted) red line indicates the limit for the 0-lepton (2-lepton) analysis alone [20].

\section{7. $A \rightarrow Z h$}

The search for a CP-odd Higgs boson $A$, decaying into a $Z$ boson and a CP-even Higgs boson $h$ with a mass of $125 \mathrm{GeV}$, considers decays of $Z$ bosons to pairs of charged leptons, $Z \rightarrow e^{+} e^{-}$and $Z \rightarrow \mu^{+} \mu^{-}$(2-lepton analysis), or neutrinos, $Z \rightarrow v \bar{v}$ (0-lepton analysis) [20]. Decays of $h$ bosons to pairs of $b$ quarks are studied, in which the resulting hadronic jets are expected to be either resolved or merged in the detector. Although the analysis is optimized for production via gluon-gluon fusion, it has been found to provide significant efficiency for $b$-quark associated production as well. Therefore, the selection is extended to events with one or more additional $b$-tagged jets. No evidence for the production of an $A$ boson in these channels is observed. The estimated 95\% CL upper limits on the product of production cross sections and branching ratios, $\sigma(p p \rightarrow A) \cdot B R(A \rightarrow$ $Z h) \cdot B R(h \rightarrow b \bar{b})$, are in the range of [4.0,0.017] pb $([6.9,0.026] \mathrm{pb})$ for $m_{A}=[220,2000] \mathrm{GeV}$ assuming exclusively gluon-gluon fusion ( $b$-quark-associated) production (Figure 9). The results are also interpreted in the context of $2 \mathrm{HDM}$ scenarios.

\section{8. $H^{+} \rightarrow t b$}

Charged Higgs bosons, heavier than the top quark and decaying via $H^{ \pm} \rightarrow t b$, are searched for in protonproton collisions measured with the ATLAS experiment at $\sqrt{s}=8 \mathrm{TeV}$, corresponding to an integrated luminosity of $8 \mathrm{fb}^{-1}[21]$. The production of a charged Higgs

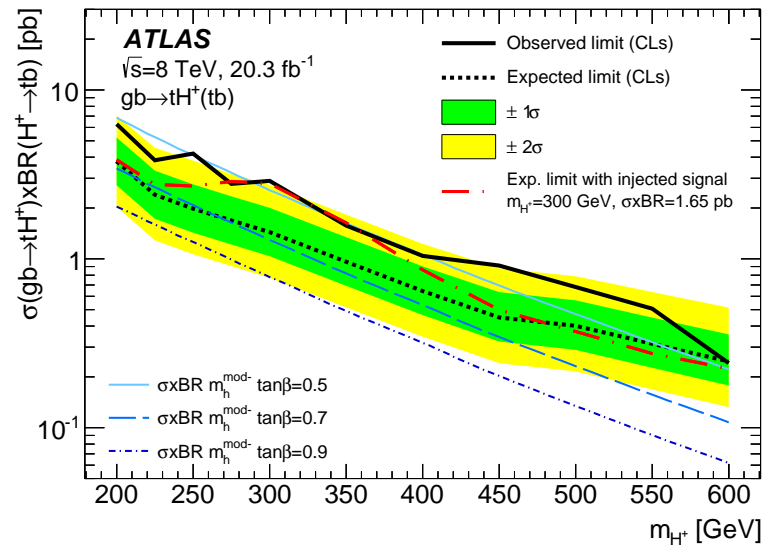

Figure 10: 95\% CL upper limits on the production of $H^{+} \rightarrow t b$ in association with a top quark, as a function of the hypothesized Higgs boson mass [21]. The red dash-dotted line shows the expected limit obtained in the case where a simulated signal is injected at $m_{H^{+}}=300$ $\mathrm{GeV}$ with a production cross section times branching ratio of $1.65 \mathrm{pb}$ (which corresponds to the best-fit signal strength at that mass hypothesis). Theory predictions are also shown for three representative values of $\tan \beta$ in the $m_{H}^{\text {mod- }}$ benchmark scenario of the MSSM.

boson in association with a top quark, $g b \rightarrow t H^{ \pm}$, is explored in the mass range from 200 to $600 \mathrm{GeV}$ using multi-jet final states with one electron or muon. An excess of events above the background-only hypothesis is observed across a wide mass range, amounting to up to 2.4 standard deviations. Upper limits are set (Figure 10 on the $g b \rightarrow t H^{ \pm}$production cross section times the branching ratio $\mathrm{BR}\left(H^{ \pm} \rightarrow t b\right)$.

\section{9. $t H^{+} \rightarrow(q q b) \tau v$}

Charged Higgs bosons, produced in association with a single top quark and decaying via $H^{ \pm} \rightarrow \tau v$, are searched for [22] in events containing a hadronic $\tau$ decay, large missing transverse momentum, a hadronically decaying top quark and no high- $p_{\mathrm{T}}$ electrons and muons. The spectrum of the transverse mass, $m_{\mathrm{T}}\left(E_{\mathrm{T}}^{\mathrm{miss}}, \tau_{\mathrm{had}}\right)$, defined by the missing transverse momentum and the visible products of the $\tau$ decay, shows no excess of the observed data above the expected background from SM processes. The statistical analysis leads to $95 \%$ CL upper limits on the production cross section times branching ratio $\sigma\left(p p \rightarrow[b] t H^{ \pm}\right)$. $B R\left(H^{ \pm} \rightarrow \tau v\right)$, for charged Higgs boson masses ranging from 200 to $2000 \mathrm{GeV}$. Such an estimation of upper limits, in the context of the hMSSM scenario [23], is shown in Figure 11 . 


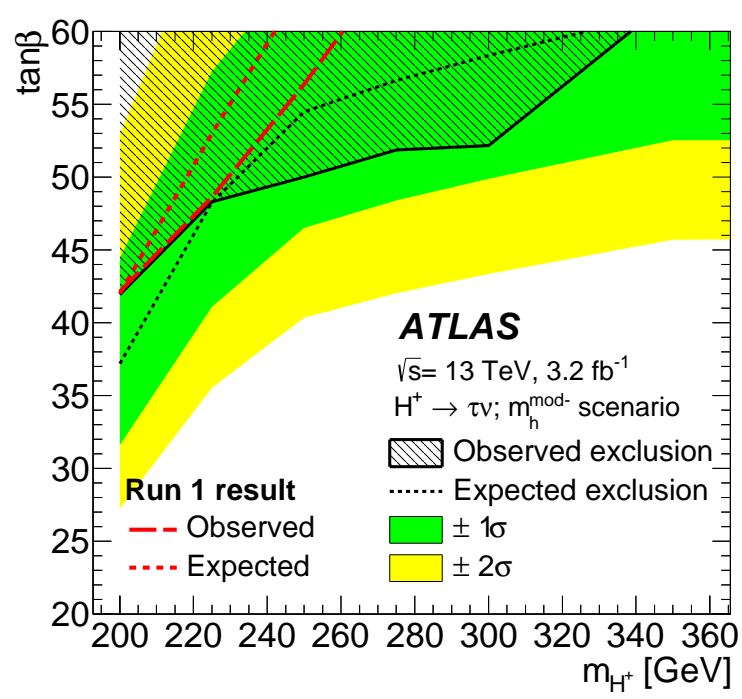

Figure 11: $95 \% \mathrm{CL}$ exclusion limits on $\tan \beta$, as a function of the hypothesized Higgs boson mass, shown in the context of the $m_{H}^{\bmod -}$ benchmark scenario of the hMSSM, for regions in which reliable theoretical calculations exist $(\tan \beta \leq 60)[22]$. For comparison, the red curves show the observed and expected exclusion limits from LHC Run-I analyses of $p p$ collisions recorded at $\sqrt{s}=8 \mathrm{TeV}$ by ATLAS.

\section{Conclusions}

A wide variety of searches for heavy Higgs bosons are carried out in the ATLAS experiment. The latest results show that the observed data are consistent with the SM prediction in most cases. The search in the $H \rightarrow \gamma \gamma$ decay mode reveals a deviation from the background-only hypothesis in the di-photon invariant mass spectrum, at $\sim 750 \mathrm{GeV}$, which corresponds to a local (global) significance of $3.9 \sigma(2.1 \sigma)$. Further information will be available with the analysis of subsequent data which are expected to improve the sensitivity and allow investigation in even higher mass regions.

\section{References}

[1] ATLAS Collaboration, JINST 3 (2008) S08003

[2] CMS Collaboration, JINST 3 (2008) S08004

[3] L. Evans and P. Bryant (eds.), JINST 3 (2008) S08001

[4] ATLAS Collaboration, Phys. Lett. B 716 (2012) 1

[5] CMS Collaboration, Phys. Lett. B 716 (2012) 30

[6] S. L. Glashow, Nucl. Phys. 22 (1961) 579

[7] S. Weinberg, Phys. Rev. Lett. $19(1967) 1264$

[8] A. Salam, Nobel Symposium 1968, ed. N. Svartholm.

[9] G. C. Branco et. al., Phys. Rept. 516 (2012) 1

[10] M. Carena et. al., Eur. Phys. J. C73 (2013) (9) 2552

[11] ATLAS Collaboration, ATLAS-CONF-2016-016, https://cds.cern.ch/record/2141005

[12] ATLAS Collaboration, ATLAS-CONF-2015-071, https://cds.cern.ch/record/2114843
[13] ATLAS Collaboration, ATLAS-CONF-2015-068, https://cds.cern.ch/record/2114840

[14] ATLAS Collaboration, ATLAS-CONF-2015-059, https://cds.cern.ch/record/2114825

[15] ATLAS Collaboration, ATLAS-CONF-2016-012, https://cds.cern.ch/record/2140833

[16] ATLAS Collaboration, ATLAS-CONF-2016-021, https://cds.cern.ch/record/2147445

[17] ATLAS Collaboration, JHEP 09 (2016) 1

[18] ATLAS Collaboration, ATLAS-CONF-2016-010, https://cds.cern.ch/record/2139795

[19] ATLAS Collaboration, ATLAS-CONF-2015-061, https://cds.cern.ch/record/2114827

[20] ATLAS Collaboration, ATLAS-CONF-2016-015, https://cds.cern.ch/record/2141003

[21] ATLAS Collaboration, JHEP 03 (2016) 127

[22] ATLAS Collaboration, Phys. Lett. B759 (2016) 555

[23] A. Djouadi et. al., Eur. Phys. J. C73 (2013) 2650 